\title{
Critical lower limb ischemia, hybrid procedures and the need for special hybrid theatres
}

Volume 8 Issue 4 - 2017

\section{Introduction}

Hybrid procedures involve revascularisation processes that are a mixture of endovascular techniques and open vascular surgery. Vascular surgeons use surgical and endovascular procedures simultaneously to treat multilevel disease in patients. Hybrid procedures are preferred in patients with multiple co-morbidities as the procedures are less invasive and associated with a reduced inhospital stay, lower mortality and morbidity. ${ }^{1-3}$ This paper is an Audit where the percentage of patients who underwent hybrid vascular procedures for lower limb ischemia in three months of three years in Aberdeen Royal Infirmary Hospital were looked at and the results discussed to emphasise the need for a new Hybrid Vascular Theatre at Aberdeen Royal Infirmary Hospital.

\section{Hybrid procedures}

Endovascular and surgical procedures were first combined and conducted in the 1970s. ${ }^{4}$ Hybrid procedures have been reported to have primary success rates ranging from $93 \%$ to $100 \%{ }^{2,5,6}$ Outcomes of hybrid endovascular procedures are comparable to pure surgical interventions. Hybrid procedures have patency rates of 60 to 90 percent and are associated with lower morbidity and mortality and shorter hospital and intensive care stay. ${ }^{1,2,5,6}$ However there are reports suggesting higher morbidity rates in hybrid endovascular procedures when compared to pure endovascular interventions. ${ }^{2}$ The number of patients who received revascularisation using hybrid procedures has increased in the last 10 years and accounts for 5 to 20 percent of cases per year. ${ }^{2,7}$

\section{Hybrid procedures in lower limb ischemia}

Hybrid procedures can be divided into three groups:

a. Open vascular surgery and endovascular intervention distal to the site of open reconstruction

b. Open vascular repair and endovascular procedure proximal to the site of open surgery.

c. Open vascular surgery and endovascular procedures both at the distal and proximal sites of open procedures. ${ }^{9}$

The iliac, femoral and infra-popliteal artery sites are often used for revascularisation in people with multilevel critical limb ischemia. A patient may need surgical treatment at certain sites and endovascular at others when ischemia is at many levels. In hybrid endovascular theatres both can be carried out at the same time one after another during one procedure. ${ }^{3}$ The common site of atherosclerosis in lower limb vessels is at the site of common femoral artery bifurcation. Atherosclerotic lesions at this site create many endovascular treatment challenges. The deep and superficial femoral artery origins are compromised by an atherosclerotic lesion at this site, if a balloon angioplasty is attempted at the site of the common femoral artery and a guide wire is inserted into the superficial femoral artery, the deep formal artery will then become occluded.

\author{
Lehri Meher, Renwick Bryce \\ Vascular Surgery Department at Aberdeen Royal Infirmary \\ Hospital, UK
}

Correspondence: Lehri Meher,Vascular Surgery Department at Aberdeen Royal Infirmary Hospital, UK, Email meher.lehri@nhs.net

Received: July 27, 2017| Published: August 18, 2017

Common femoral arteries are always under continuous movement and there is a danger of stents fracturing as a result of that. Once the stents are inserted access to common femoral artery then becomes very challenging. Common femoral artery can be accessed with ease surgically therefore endarterectomy is often the preferred choice of treatment. ${ }^{8}$ In the past common femoral artery bifurcation lesions were treated with peripheral Trans luminal angioplasty. Patients would then be discharged from the hospital and then come back for a bypass or an endarterectomy procedure. Today hybrid procedures are becoming increasingly popular and both procedures can be carried out together in one surgery.

\section{A. Trans-Atlantic Inter Society Consensus on the Management of Peripheral Arterial Disease (TASC II) document}

A document called the trans-Atlantic inter-society consensus document on management of peripheral arterial disease (TASC) was published in January 2000 for management of peripheral arterial disease. This document was a collaberative effort of fourteen vascular, medical, radiology, cardiology and cardiovascular societies belonging to North America and Europe. ${ }^{10-12}$ This document was revised and developed to include the consensus of Africa, Asia, Australia, Europe and North America in 2004. The new document helped abbreviate the diagnosis and management plans for peripheral arterial disease. This document is used to make decisions about management plans for complex lower limb ischemia cases.

\section{Complications of hybrid procedures}

Limitations of hybrid procedures include the following:

a. Increased time of operation.

b. Longer anaesthetic time and the use of general anesthesia in hybrid procedures. In procedures performed in steps, endovascular procedures may only require local anesthesia.

c. Longer groin wound exposure time in hybrid procedures. Increasing the risk of infections. ${ }^{3}$ 


\section{Benefits of Hybrid procedures}

The technical success of the interventional procedures was enhanced by hybrid procedures. Hybrid procedures have the following advantages:

i. Reduced rates of bleeding.

ii. Lower risks of arterial damage.

iii. Accurate localisation and identification of lesions in arteries. Enabling the treatment of significant lesions in the arteries.

\section{Audit}

X-ray MDT meetings between vascular surgeons, interventional radiologists and vascular laboratory technicians happen every Thursday morning before the ward rounds in Aberdeen Royal Infirmary Hospital. In this meeting a list of clinical cases including emergency cases are discussed to decide on the best form of management for each case. There are five possible outcomes for each case discussed:

a. Vascular surgery.

b. Endovascular Procedure.

c. Hybrid Procedure.

d. Conservative management.

e. The need for further investigations.

Cases discussed include: upper limb cases, lower limb cases and thoracic and abdominal vessels. The Vascular Surgery Team in Aberdeen Royal Infirmary also holds separate Aneurysm meetings every Tuesday morning with interventional radiologists, vascular surgeons and vascular laboratory technicians from Aberdeen Royal Infirmary Hospital and Raigmore hospital to discuss Aneurysm cases. The outcomes from these meetings are one of the following:
i. Vascular surgery
ii. Hybrid Procedure
iii. Conservative
iv. The need for further investigations

\section{Method}

For this Audit I looked at lower limb ischaemia cases discussed in $\mathrm{x}$-ray MDT meetings to work out the number of cases that had hybrid outcomes. I chose the cases discussed in the months of January, February and March in 2015, 2016 and 2017.

\section{Results}

Table $1 \& 2$ shows the outcome of the $x$-ray MDT meetings between the months January, February and March for the years 2015, 2016 and 2017. Eight to ten percent of the cases discussed in x-ray MDT meetings required hybrid procedures in the months January, February and March for the years 2015, 2016 and 2017. The percentage of cases in those months and those years requiring hybrid procedures were variable. These cases were purely lower limb ischaemia cases. The actual percent of hybrid cases per anum cannot be worked out based purely on these findings and numbers as the number of hybrid cases vary per anum. These numbers exclude upper limb ischaemia and aneurysm cases. The general trend in vascular surgery has been an increase in the number of hybrid procedures per anum. ${ }^{3}$

Table I It shows the outcomes of X-ray MDT meetings for the months January, February and March and the years 2015,2016 and 2017

\begin{tabular}{lllllll} 
Year & Vascular Surgery & Endovascular & Hybrid & Conservative & Further Scans & Total Cases \\
\hline 2015 & 19 & 45 & 7 & 13 & 1 & 85 \\
2016 & 14 & 26 & 8 & 25 & 7 & 80 \\
2017 & 21 & 30 & 6 & 5 & 4 & 66 \\
\hline
\end{tabular}

Table 2 It shows the percentage of cases requiring vascular surgery, endovascular, hybrid procedures, conservative treatments and further scans in the months January, February and March for the years 2015, 2016 and 2017

\begin{tabular}{llllll}
\hline Year & Vascular Surgery & Endovascular & Hybrid & Conservative & Further Scans \\
\hline 2015 & $22 \%$ & $53 \%$ & $8 \%$ & $15 \%$ & $1 \%$ \\
2016 & $17.50 \%$ & $32 \%$ & $10 \%$ & $31.25 \%$ & $8.75 \%$ \\
2017 & $31.80 \%$ & $45 \%$ & $9 \%$ & $7.50 \%$ & $6 \%$ \\
\hline
\end{tabular}

\section{Discussion}

Multilevel critical limb ischaemia often requires a combination of endovascular and surgical interventions. ${ }^{3}$ Roughly five percent of the lower chronic limb ischemia cases discussed in $\mathrm{x}$-ray MDT meetings between the months January and March for the years 2015, 2016, 2017 were hybrid procedures. These hybrid cases would increase in number if hybrid procedures involving EVARs, FEVARs and TEVARs as well as upper limb ischemia are also considered; emphasising the need for building a special hybrid theatre in ARI. ARI currently has a small procedures room especially dedicated to hybrid vascular procedures.

\section{Hybrid procedure theatres}

A hybrid theatre incorporates the equipment and facilities of both a surgical theatre and an interventional radiology suite,$^{14}$ allowing imaging during and after operation. ${ }^{13}$ Interventional radiology suites are not suited for surgical interventions for the following reasons:
i. Lack of space for an anaesthetic machine
ii. Lack of sterile environment
iii. Lack of positive pressure air change and air cycling iv. Lack of space for interventional radiology nurses and surgical
nurses ${ }^{13}$
v. Lack of appropriate lighting
vi. Lack of facilities for suctioning

Operating theatres are equipped for quick decontamination when required to maintain a sterile environment. Imaging equipment that are portable increase the risk of contamination of a sterile environment. ${ }^{13}$

\section{The need for a Hybrid procedure theatre in Aberdeen Royal Infirmary Hospital}

The general trend in vascular surgery is an increase in the number 
of hybrid cases. At Aberdeen Royal Infirmary Hospital hybrid cases happen in a procedures room in the radiology department. This room has an aneasthetic machine and a $\mathrm{C}$-arm radiology machine. The surgical team has it's own trained nursing staff and interventional radiology has it's own. This room does not have enough space to accommodate both the teams. It lacks the following:

i. Lack of positive pressure air change and air cycling

ii. Inappropriate lighting facilities.

iii. Lack of space to accommodate both the surgical and interventional radiology teams

iv. Lack of space increases the risk of high dose radiation exposure to the interventional radiology, the surgical teams as well as the patient.

As was highlighted earlier, hybrid procedures cases are time consuming and the longer a case takes the increased the risk of infection. The above factors increase the risk of infection in hybrid cases and necessitates the need for a special hybrid theatre.

\section{Conclusion}

As Aberdeen Royal Infirmary is the main hospital in the north of Scotland that offers hybrid vascular surgical procedures to the Islands around it as well as to the patients in Aberdeen there is a dire need for a bigger specially designed hybrid theatre that offers quality hybrid interventions to patients with complex vascular cases.

\section{Acknowledgments}

None.

\section{Conflicts of interest}

Authors declare that there is no conflict of interest.

\section{References}

1. Piazza M, Ricotta JJ, Bower TC. Iliac artery stenting combined with open femoral endarterectomy is as effective as open surgical reconstruction for severe iliac and common femoral occlusive disease. J Vasc Surg. 2011;54(2):402-411.
2. Dosluoglu HH, Lall P, Cherr GS, et al. Role of simple and complex hybrid revascularization procedures for symptomatic lower extremity occlusive disease. J Vasc Surg. 2010;51(6):1425-1435.

3. PS Aho, M Venermo. Hybrid procedures as a novel technique in the treatment of critical limb ischaemia. Scand J Surg. 2012;101(2):107-113.

4. Porter JM, Eidemiller LR, Dotter CT, et al. Combined arterial dilatation and femorofemoral bypass for limb salvage. Surg Gynecol Obstet. 1973;137(3):409-412.

5. Nelson PR, Powell RJ, Schermerhorn ML. Early results of external iliac artery stenting combined with common femoral artery endarterectomy. J Vasc Surg. 2002;35(6):1107-1113.

6. Simó G, Banga P, Darabos G, et al. Stent-assisted remote iliac artery endarterectomy: an alternative approach to treating combined external iliac and common femoral artery disease. Eur J Vasc Endovasc Surg. 2011;42(5):648-655.

7. Kashyap VS, Pavkov ML, Bena JF, et al. The management of severe aortoiliac occlusive disease: endovascular therapy rivals open reconstruction. J Vasc Surg. 2008;48(6):1451-1457.

8. Kang JL, Patel VI, Conrad MF, et al. Common femoral artery occlusive disease: contemporary results following surgical endarterectomy. $J$ Vasc Surg. 2008;48(4):872-877.

9. Antoniou GA, Sfyroeras GS, Karathanos C, et al. Hybrid endovascular and open treatment for severe multilevel lower extremity and open treatment for severe multilevel aarterial disease. Eur J Vasc Endovasc Surg. 2009;38(5):616-622.

10. Management of peripheral arterial disease (PAD). Trans Atlantic InterSociety Consensus (TASC). Eur J Vasc Endovasc Surg. 2000;19(Suppl A):S1-250.

11. TASC. Management of Peripheral Arterial Disease (PAD) Trans Atlantic Intersociety Consensus (TASC). J Vasc Surg. 2000;31(1 part 2):S1287.

12. TASC. Management of peripheral arterial disease (PAD). Trans Atlantic Inter-Society Consensus (TASC). Int Angiol. 2000;19(1 Suppl 1):1-304.

13. Sikkink C, Reijnen M, Zeebregts C. The creation of the optimal dedicated endovascular suite. Eur J Endovasc Surg. 2008;35:198-204.

14. Urbanowicz J, Taylor G. Hybrid OR: Is it in your future? 